\title{
Predictive analysis of criterial yield during travelling wire electrochemical discharge machining of Hylam based composites
}

\author{
Mitra, N.S. ${ }^{a,}{ }^{*}$, Doloi, B. ${ }^{b}$, Bhattacharyya, B. ${ }^{b}$

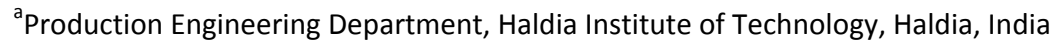 \\ ${ }^{b}$ Production Engineering Department, Jadavpur University, Kolkata, India
}

\begin{abstract}
A B S T R A C T
Travelling wire electrochemical discharge machining (TW-ECDM) has great potential for machining advanced non-conducting materials such as zirconia, alumina, silicon nitride, diamond glass, rubies and composites such as FRP etc. Composite materials possess higher strength, stiffness, and fatigue limits which enable structural design more flexible than with conventional metals. Over recent years precision machining of composite materials has gained in importance. The presented research paper includes a description of an indigenously developed TW-ECDM set-up for performing experiments on composite materials such as fibre reinforced plastic. This paper also presents analyses of machining parameters such as material removal rate and radial overcut for different input parameters such as pulse on time, frequency of power supply, applied voltage, concentration of electrolyte and wire feed rate. Taguchi method-based optimization analysis was also done for achieving minimum radial overcut and maximum material removal rate during the cuttings of grooves on Hylam based fibre reinforced composites. Multiple regression models were also established for both material removal rate and radial overcut by considering the more important process parameters for cutting grooves on Hylam based fibre reinforced composites. Finally, a back propagation neural network was applied for predicting the responses and those predictions are compared with the experimental results.
\end{abstract}

\section{ARTICLE INFO}

Keywords:

TW-ECDM

Groove cutting

Fibre reinforced composites

Taguchi method

Artificial neural nets

*Corresponding author: nsmitra@rediffmail.com (Mitra, N.S.)

Article history:

Received 2 March 2014

Revised 24 March 2015

Accepted 26 March 2015 


\title{
Predikcijska analiza kriterijev učinkovitosti med obdelavo kompozitov Hylam s pomočjo elektrokemične erozijske obdelave s potujočo žico
}

\author{
Mitra, N.S. ${ }^{a}{ }^{\text {* }}$, Doloi, B. ${ }^{b}$, Bhattacharyya, B. ${ }^{b}$

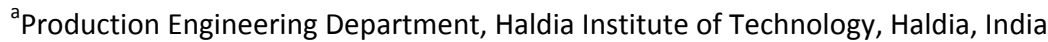 \\ ${ }^{\mathrm{b}}$ Production Engineering Department, Jadavpur University, Kolkata, India
}

\section{POVZETEK}

Elektrokemična erozijska obdelava s potujočo žico (TW-ECDM) ima velike možnosti obdelave naprednih neprevodnih materialov, kot so cirkonij, aluminijev oksid, silicijev nitrid, diamantno steklo in tudi kompozitov, kot je npr. FRP. Kompozitni materiali imajo visoko čvrstost, togost in mejo utrujenosti materiala, kar omogoča višjo prilagodljivost pri načrtovanju izdelkov kot pa pri običajnih materialih. $\mathrm{V}$ zadnjih letih je natančna obdelava kompozitnih materialov vse pomembnejša. Pričujoča raziskava vključuje opis doma razvite obdelave TW-ECDM, ki je bila preizkušena za obdelavo kompozitnih materialov, kot je npr. z vlakni okrepljena plastika. Prispevek opisuje tudi analizo obdelovalnih parametrov, kot so stopnja odvzema materiala in overcut, in sicer za različne vhodne parametre, kot so čas trajanja impulza, frekvenca električnega vira, velikost napetosti, koncentracija elektrolita in podajanje žice. Da bi dosegli minimalni radialni overcut in čim večjo stopnjo odvzema materiala pri rezanju utora na kompozitu Hylam, učvrščenem z vlakni, je bila narejena optimizacija s pomočjo metode Taguchi. Prav tako smo s pomočjo multipla regresijske metode razvili modele za stopnjo odvzema materiala in radialni overcut, upoštevajoč najbolj pomembne procesne parameter. $\mathrm{Na}$ koncu je bila aplicirana še umetna nevronska mreža z vzvratnim razširjanjem za napovedovanje odzivov, ki smo jih primerjali tudi z eksperimentalnimi rezultati.
\end{abstract}

(C) 2015 PEI, University of Maribor. All rights reserved.

\section{PODATKI O ČLANKU}

Ključne besede:

TW-ECDM

Rezanje utorov

Z vlakni okrepljeni kompoziti

Metoda Taguchi

Umetne nevronske mreže

*Kontaktna oseba:

nsmitra@rediffmail.com

(Mitra, N.S.)

Zgodovina članka:

Prejet 2. marca 2014

Popravljen 24. marca 2015

Sprejet 26. marca 2015 


\section{References}

[1] Bhattacharyya, B., Doloi, B.N., Sorkhel, S.K. (1999). Experimental investigations into electrochemical discharge machining (ECDM) of non-conductive ceramic materials, Journal of Materials Processing Technology, Vol. 95, No. 1-3, 145-154, doi: 10.1016/S0924-0136(99)00318-0.

[2] Basak, I., Ghosh, A. (1997). A mechanism of material removal in electrochemical discharge machining: A theoretical model and experimental verification, Journal of Materials Processing Technology, Vol. 71, No. 3, 350-359, doi: 10.1016/S0924-0136(97)00097-6.

[3] Jain, V.K., Adhikary, S. (2008). On the mechanism of material removal in electrochemical spark machining of quartz under different polarity conditions, Journal of Materials Processing Technology, Vol. 200, No. 1-3, 460-470, doi: 10.1016/j.jmatprotec.2007.08.071.

[4] Chak, S.K., Rao, P.V. (2007). Trepanning of $\mathrm{Al}_{2} \mathrm{O}_{3}$ by electro-chemical discharge machining (ECDM) process using abrasive electrode with pulsed DC supply, International Journal of Machine Tools and Manufacture, Vol. 47, No. 14, 2061-2070, doi: 10.1016/j.ijmachtools.2007.05.009.

[5] Jain, V.K., Rao, P.S., Choudhary, S.K., Rajurkar, K.P. (1991). Experimental investigations into travelin wire electrochemical spark machining (TW-ECSM) of composites, Journal of Manufacturing Science and Engineering, Vol. 113, No. 1, 75-84, doi: $10.1115 / 1.2899625$.

[6] Peng, W.Y., Liao, Y.S. (2004). Study of electrochemical discharge machining technology for slicing non-conductive brittle materials, Journal of Materials Processing Technology, Vol. 149, No. 1-3, 363-369, doi: 10.1016/j. jmatprotec.2003.11.054.

[7] Tandon, S., Jain, V.K., Kumar, P., Rajurkar, K.P. (1990). Investigation into machining of composites, Precision Engineering, Vol. 12, No. 4, 227-238, doi: 10.1016/0141-6359(90)90065-7.

[8] Jain, V.K., Choudhury, S.K., Ramesh, K.M. (2002). On the machining of alumina and glass, International Journal of Machine Tools and Manufacture, Vol. 42, No. 11, 1269-1276, doi: 10.1016/S0032-3861(02)00241-0.

[9] Kulkarni, A., Sharan, R., Lal, G.K. (2002). An experimental study of discharge mechanism in electrochemical discharge machining, International Journal of Machine Tools and Manufacture, Vol. 42, No. 10, 1121-1127, doi: 10.1016/S0890-6955(02)00058-5.

[10] Wüthrich, R., Hof, L.A. (2006). The gas film in spark assisted chemical engraving (SACE) - A key element for micro-machining applications, International Journal of Machine Tools and Manufacture, Vol. 46., No. 7-8, 828835, doi: 10.1016/j.ijmachtools.2005.07.029.

[11] Mediliyegedara, T.K.K.R., De Silva, A.K.M., Harrison, D.K., McGeough, J.A. (2004). An intelligent pulse classification system for electrochemical discharge machining (ECDM) - A preliminary study, Journal of Material Processing Technology, Vol. 149, No. 1-3, 499-503, doi: 10.1016/j.jmatprotec.2004.04.002.

[12] Bhondwe, K.L., Yadava, V., Kathiresan, G. (2006). Finite element prediction of material removal rate due to electro-chemical spark machining, International Journal of Machine Tools and Manufacture, Vol. 46, No. 14, 16991706, doi: 10.1016/j.ijmachtools.2005.12.005.

[13] Manna, A., Kundal, A. (2011). Micromachining of nonconductive $\mathrm{Al}_{2} \mathrm{O}_{3}$ ceramic on developed TW-ECSM setup, International Journal of Manufacturing, Materials and Mechanical Engineering, Vol. 1, No. 2, 46-55, doi: 10.4018/ijmmme.2011040103

[14] Mallick, B., Sarkar, B.R., Doloi, B., Bhattacharyya, B. (2013). Parametric analysis of travelling wire electrochemical discharge machining process, Reason - A Technical Journal, Vol. 12, 93-101.

[15] Jawalkar, C.S., Sharma, A.K., Kumar, P. (2012). Micromachining with ECDM: Research potentials and experimental investigations, International Journal of Mechanical and Aerospace Engineering, Vol. 6, 7-12.

[16] Manna, A., Narang, V. (2012). An experimental investigation during micro-machining of e-glass fibre epoxy composite on developed electrochemical spark machining setup, International Journal of Manufacturing, Materials and Mechanical Engineering, Vol. 2, No. 2, 46-60, doi: 10.4018/ijmmme.2012040103.

[17] Cao, X.D., Kim, B.H., Chu, C.N. (2013). Hybrid micromachining of glass using ECDM and micro grinding, International Journal of Precision Engineering and Manufacturing, Vol. 14, No. 1, 5-10, doi: 10.1007/s12541-013-0001-6.

[18] Mitra, N.S, Doloi, B., Bhattacharyya, B. (2014). Analysis of traveling wire electrochemical discharge machining of Hylam based composites by Taguchi method, International Journal of Research in Engineering and Technology, Vol. 2, No. 2, 223-236.

[19] Manna, A., Kundal, A. (2015). An experimental investigation on fabricated TW-ECSM setup during micro slicing of nonconductive ceramic, The International Journal of Advanced Manufacturing Technology, Vol. 76, No. 1-4, 2937, doi: $10.1007 / \mathrm{s} 00170-013-5145-0$.

[20] Phadke, M.S. (1989). Quality engineering using robust design, Prentice Hall, Englewood Cliffs, New Jersey, USA. 